\title{
Design of 8-Bit ALU Design using GDI Techniques with Less Power and Delay
}

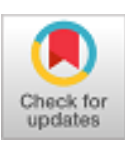

\author{
Harshmaniyadav Uday Panwar,
}

\begin{abstract}
Arithmetic Logic Unit (ALU) is a substantial fragment of microchip. Cutting-edge computerized processors, legitimate and math activity accomplishes making use of ALU. This paper depicts an 8-Bit ALU operating with a lowest power 11-Transistor Full Adder (11-T FA) and Gate dispersion input (GDI) centered MUX. All structures were simulated using Tanner EDA software version-15 with 32 nanometer BSIM4 innovation. Execution examinations were furnished as for voltage, power, postponement and power delay item. In this paper 8-bit $A L U$ operated in subthreshold region, selected $0.7 V_{D D}$ for maintain the both power as well as delay. In 8-bit ALU of GDI proposed model, less than $82 \%$ power consumption reduced as compare with CMOS 8-Bit ALU due to voltage level improvement.
\end{abstract}

Keyword--- ALU (Arithmetic logic unit), FA (Full Adder), GDI (Gate diffusion input), MUX (Multiplexer)

\section{INTRODUCTION}

The utilization of VLSI innovation has long-drawn-out to that level here a massive amount of transistors be able to be actualized in a solitary Bit. Complementary Metal Oxide Semiconductor (CMOS) remained the spine in blended sign as this one's diminishing force offers great blend segment for modest and computerized structure. The supports of intensity utilization in CMOS circuits are dynamic power $\left(\mathrm{P}_{\mathrm{d}}\right)$, short out power $\left(\mathrm{P}_{\mathrm{sc}}\right)$ and static power $\left(\mathrm{P}_{\mathrm{s}}\right)$. Along these lines, the complete power utilization $\left(\mathrm{P}_{t}\right)$ is

$\mathrm{P}_{\mathrm{t}}=\mathrm{P}_{\mathrm{d}}+\mathrm{P}_{\mathrm{sc}}+\mathrm{P}_{\mathrm{s}}$

$P_{d}$ expends because of capacitive burden in addition to clock recurrence. $\mathrm{P}_{\mathrm{sc}}$ is brought about by short out current. $\mathrm{P}_{\mathrm{s}}$ is brought about by leakage current between the substrate and dispersion region. Expanding transistor quantityfor every chip territory plus scaling down advances have devoured additional power along these lines.

Manuscript published on November 30, 2019.

* Correspondence Author

Harshmani Yadav*, Electronics \&Amp; Communication, Sager Institute Of Research \&Amp;

Technology, Bhopal, India. Email: Theharshmani@Gmail.Com

Uday Panwar, Electronics \&Amp; Communication, Sager Institute Of Research \&Amp; Technology

Bhopal, India. Email: Panwaruday1@Gmail.Com

(C) The Authors. Published by Blue Eyes Intelligence Engineering and Sciences Publication (BEIESP). This is an open access article under the CC-BY-NC-ND license http://creativecommons.org/licenses/by-nc-nd/4.0/
The principle goal line is in the direction of decreasing the power utilization via employing unique approaches intended to improve the demonstration of Very Large Scale Integration circuits. Arithmetic Logic Unit is the segment of PC processor which accomplishes number-crunching as well as coherent activities [1]. ALU is a solely combinational rationale circuit whose yield deviates with altering information reaction.

\section{PREVIOUS WORK}

Power diminishing could be achieved at Module Level or at circuit level or at architecture level [2]. In simple switch procedure select info rationale as control rationale and permits additional info signal from gate terminal [3]. Full Adder is a fundamental structure in place of planning an ALU. Various sorts of FA planning in place of limiting force are, for example, Hybrid Full Adder (H-FA) and 10 Transistor reduced power Full Adder (10T-FA) and 11 transistor FA (11T-FA) etc. FA works in mode of ultra-low through utilization of subthreshold current then expends low power [2], [3]. Full Adder is manufactured utilizing near to the ground power XOR gate and 2:1 multiplexer. ALU configuration employing FinFET innovation has dual gates that are electrically free. This limits the intricacy of the circuit and furthermore lessens the power utilization because of diminishing the leakage current. In Fin Field Effect Transistor (FinFET) innovation "Fin" is dainty silicon that shapes the frame of the gadget [4]. Arithmetic Logic Unit plan utilizing the re-configurable rationale of Multiple Input Floating Gate - Metal Oxide Semiconductor (MIFG-MOS) transistor has numerous information that expands the effectiveness of the circuit. MIFG-MOS transistor offers low and high conditions by watching theweighted aggregate of altogether things considered. MIFG-MOS transistor diminishes the quantity of transistors and the unpredictability with the circuit in addition to improving the exhibition of the circuit limit the postponement as well as diminishes the power dissemination [5]. AT the point where channel size is scaled discouraged for organizing the circuits, Gate of metal and higher value dielectric $\mathrm{K}$ are to be advertised.

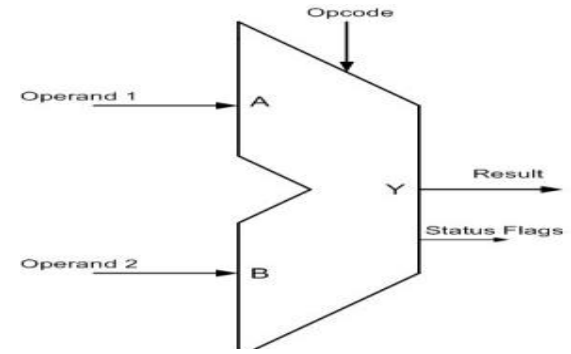

Figure 1. ALU Symbol

Published By:

Retrieval Number: D9544118419/2019@BEIESP

DOI:10.35940/ijrte.D9544.118419

Journal Website: www.ijrte.org

10083 Blue Eyes Intelligence Engineering \& Sciences Publication 


\section{Design of 8-bit ALU Design using GDI Techniques with Less Power and Delay}

\section{CIRCUIT DESIGN FOR ALU}

Arithmetic Logic Unit is the center Bit of PC/computerized processor that implements number juggling as well as consistent activity.

For example, increase, decrement, expansion and subtraction as an arithmetic unit and OR, AND, X-OR, XNOR as a Logic Unit. Arithmetic Logic Unit works through making use of Full Adder and Mux.

Table 1. Truth Table (TT) of 8-Bit ALU

\begin{tabular}{|c|c|c|c|}
\hline S2 & S1 & So & Functionality \\
\hline 0 & 0 & 0 & Operand1 | operand2 \\
\hline 0 & 0 & 1 & $\sim($ Operand1 ^ operand2) \\
\hline 0 & 1 & 0 & (Operand1 ^ operand2) \\
\hline 0 & 1 & 1 & Operand1 \& operand2 \\
\hline 1 & 0 & 0 & ++ Operand \\
\hline 1 & 0 & 1 & Operand1 + operand2 \\
\hline 1 & 1 & 0 & -- Operand \\
\hline 1 & 1 & 1 & Operand1 - operand2 \\
\hline
\end{tabular}

\section{A. 11 Transistors Full Adder Circuit Design}

11 Transistors Full Adder (11T FA) acts the fundamental utilitarian component of an ALU Structure. 11T utilized structure of FA limits the power besides diminishing the deferral. FA outlined in Figure. 2 operates on a power source $\left(V_{\mathrm{DD}}\right)$ of 0.9 voltage. Sources of information $A$ is applied to the gate terminal of NMOS-1 and PMOS-1 besides channel terminal of PMOS-2. Sources of information B is applied to NMOS-2 and PMOS-2 of gate terminal as well as the NMOS-1 of channel terminal. At the point as soon as source voltage $\left(\mathrm{V}_{\mathrm{s}}\right)$ is more noteworthy compared to Threshold Voltage $\left(\mathrm{V}_{\mathrm{TH}}\right)$, the transistor is $\mathrm{ON}$ condition and passes the sign deplete point from gate point and passes the gate voltage $\left(\mathrm{V}_{\mathrm{G}}\right)$ to the drain terminal. Hence, when i/p A is ON, then pass i/p B other way around. FA is assembling utilizing minimal power XOR gates and 2:1 mux. XOR gates give the aggregate yield and multiplexer dependable for complete (Cout).

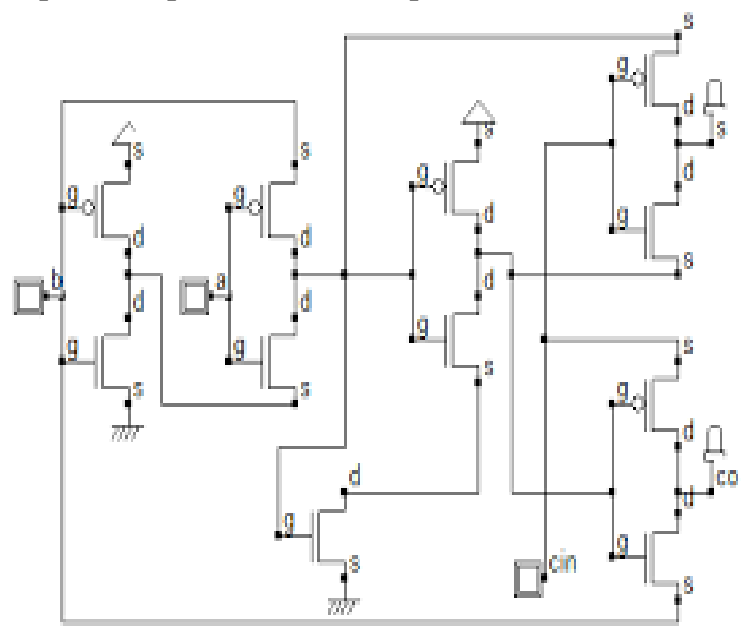

Figure 2. Schematic of 11 Transistor Full Adder

An additional NMOS-6 transistor uses sub-threshold current in ultra-low mode and devours low-control. A solid reversal district gate terminal voltage to voltage of source terminal $\left(\mathrm{V}_{\mathrm{GS}}\right)$ is greater than the voltage of Threshold level $\left(\mathrm{V}_{\mathrm{TH}}\right)$, dominant part bearers expelled from the region of the gate and minority bearers are created, at powerless reversal area
$\mathrm{V}_{\mathrm{GS}}$ is underneath than $\mathrm{V}_{\mathrm{TH}}$ less minority bearer is delivered, yet their essence produces spillagecurrent this current is called sub-threshold current. If $\mathrm{V}_{\mathrm{dd}}$ isbelow than the $\mathrm{V}_{\mathrm{th}}$, this current can be used and the circuitkeep running in ultra-low mode and utilize less power. 11T FA operate with outside NMOS-6 transistor in subthreshold mode.

\section{B. GDI established MUXs}

GDI procedure is an area effective procedure that ingests lesser power in addition to reducing the numeral of transistors required [7]. GDI techniques extends the chip configuration 'twin well' or silicon on insulator [8]. In addition to optimizing gain plus $\mathrm{V}_{\text {th }}$ of N-Type and P-Type unit, Twin-well system provides isolated optimization for ntype and p-type transistors [9]. Metal oxide semiconductor and bipolar expertise are correlated with silicon on insulator in a solitary phase. GDI practice affords more input to the cell and maintains complexity of circuit. GDI procedure resolves the issue of weak high to low switching condition of PMOS and offers a full swing on the interior point of the circuit [10]. Figure 3 portrays a GDI established 2:1 multiplexer.

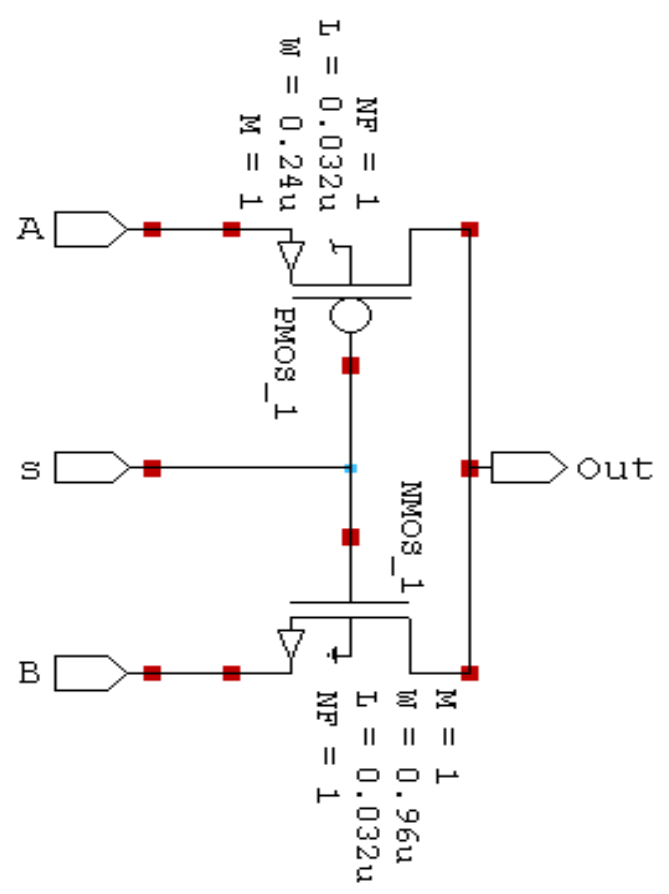

Figure 3. GDI established Mux 2

The select line $\mathrm{S}$ of mux is mutual for the input of gate terminals of NMOS-1 as well as PMOS-1. Information of A and $\mathrm{B}$ are linked towards the PMOS-1 and NMOS-1 at source terminals individually. At the point where $\mathrm{S}$ is low then and there exits the condition were PMOS-1 is in highstate and passes the information A from the terminal of source to terminal of drain. NMOS-1 is high at the point where $\mathrm{S}$ is high, while PMOS-1 low. Yield is regular for PMOS-1 and NMOS-1 channel terminals. Fig. 4 outlines the 4:1 multiplexer.

4:1 multiplexer is configured utilizing three 2: 1 multiplexer [10]. The $S_{0}$ and $S_{1}$ selection lines are filled in as an exchanging input that is responsible for the transistors $\mathrm{ON}$ and OFF conditions [11], [12].

Published By:

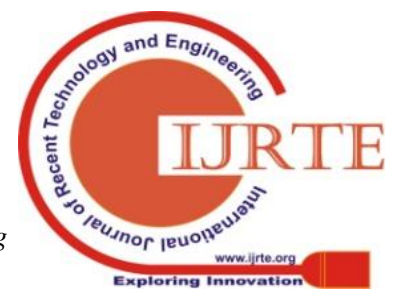




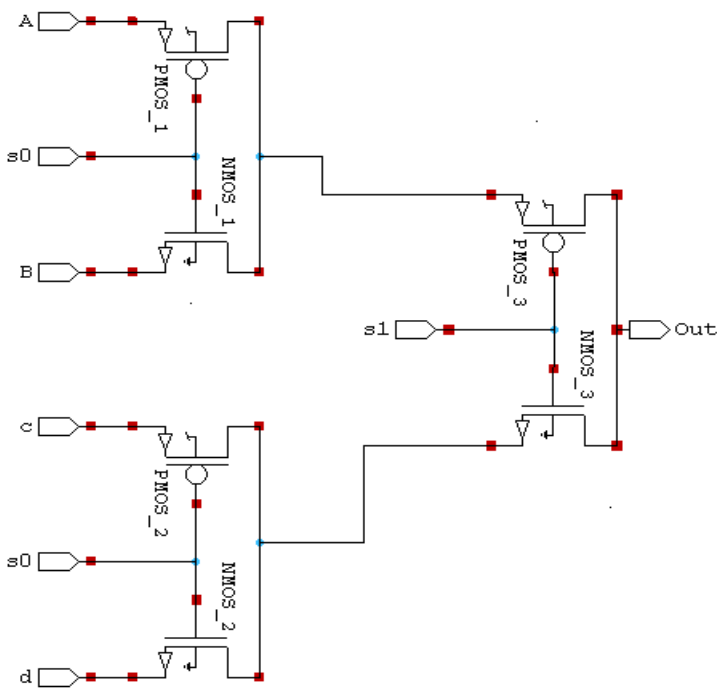

Figure 4. GDI established MUX 4

$\mathrm{S}_{0}$ is normal to enter in place of NMOS-1, NMOS-2, PMOS1 and PMOS-2 at gate terminal, $S_{1}$ is normal contribution in place of gate terminals of NMOS-3 as well as PMOS-3. Information sources remain associated with the transistor's source terminal.

\section{Plan of an 8 bit $A L U$}

FA acts as the backbones for Arithmetic Logic Unit. 8-Bit Arithmetic Logic Unit is planned utilizing 8-Bit Ripple Carry Adder [13], [14]. Ripple Carry Adder is in charge of the number juggling activity performed by ALU. Different building blocks required to structure ALU are 2:1 multiplexer and 4:1 multiplexer. Legitimate activity is performed by utilizing mux [15], [16]. Figure 5 outlines a 1Bit Arithmetic Logic Unit configuration. It utilizes two 4:1 multiplexer and one 2:1 mux and a FA.

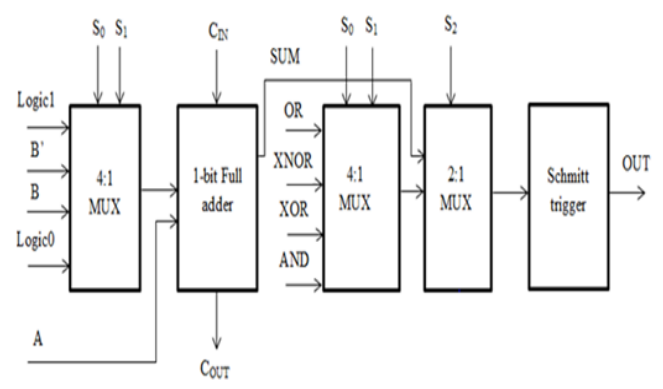

Figure 5. 1 bit ALU

Figure 6 portrays 8-Bit Arithmetic Logic Unit, Ripple carry Adder is the fundamental structure square of 8-Bit Arithmetic Logic Unit which plays out a math activity. Info A $(\mathrm{a} 0, \mathrm{a} 1, \ldots . \mathrm{a} 8)$ was applied in head contribution of Ripple carry Adder, Info B (b0, b1,...b8) was applied with the first contribution of $4: 1$ mux and goes to the second contribution of Ripple carry Adder the yield of 4:1 multiplexer and implements the math activity. Consistent activity performs through the falling mix of 4:1 multiplexer and 2:1 multiplexer.

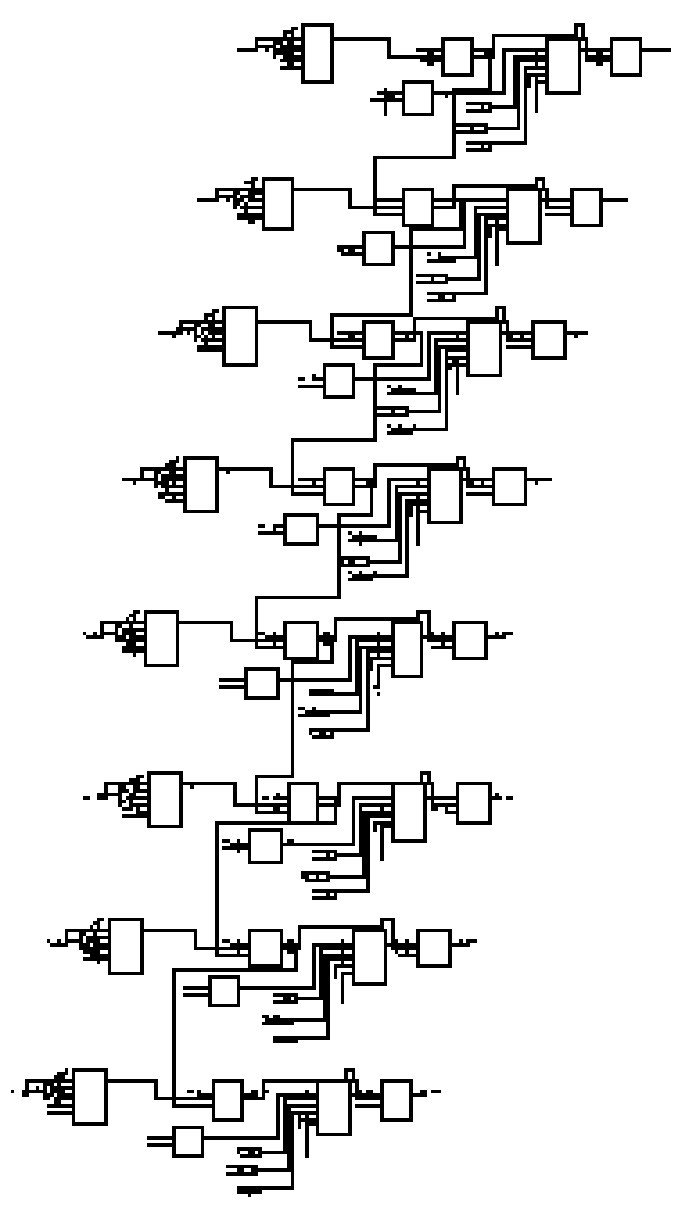

Figure 6. 8 Bit ALU

\section{RECREATION AND RESULTS}

All plan were recreated utilizing Tanner EDA apparatus Walter at progress time 32 nanosecond (ns). Figure. 7 demonstrates the graph obtained for 11-T FA, by input blend 000, 001, 010, 011, 100, 101, 110 and 111. At 000, 010 \& 110 issue were spotted. These issue are unraveled via including an additional transistor NMOS-6

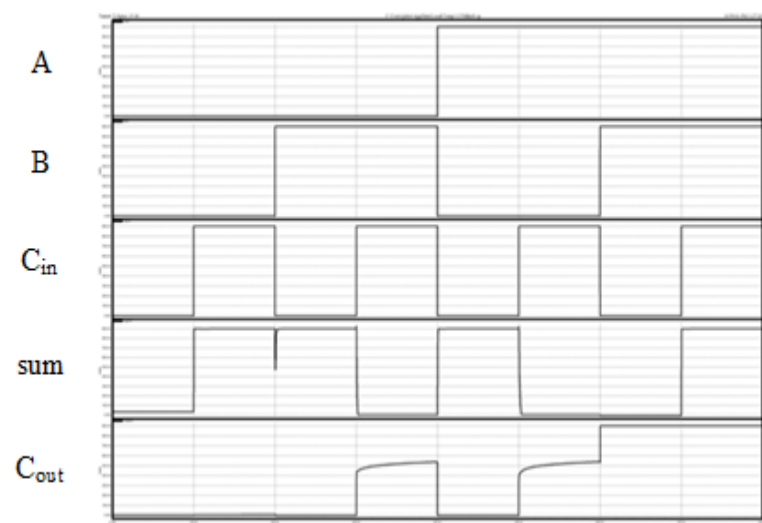

Figure 7. Wave shape obtained for $11 T$ 
Design of 8-bit ALU Design using GDI Techniques with Less Power and Delay

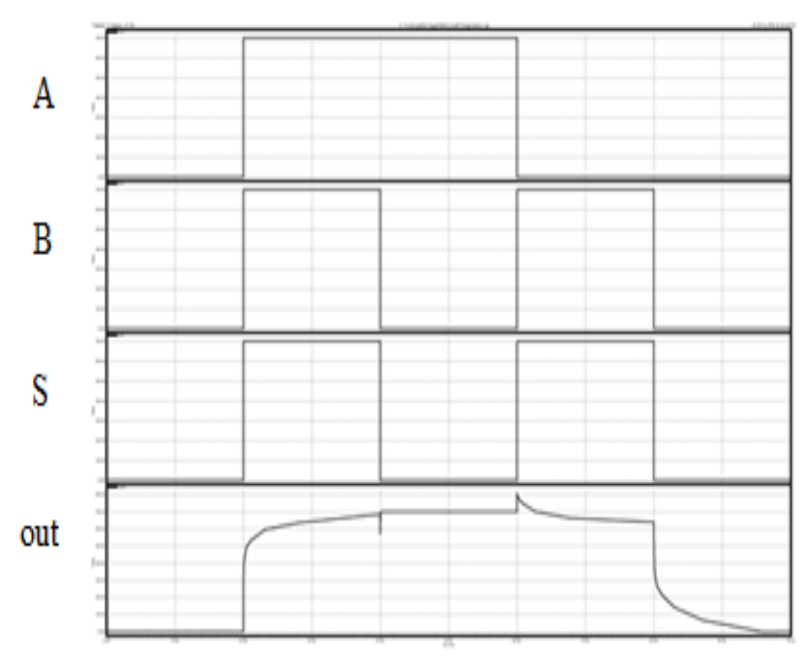

Figure 8 Wave shape of 2:1 MUX

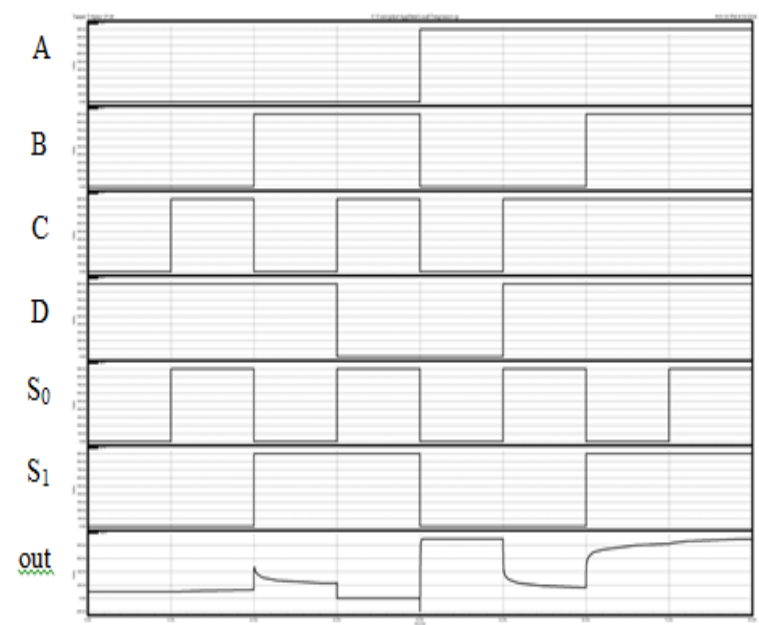

Figure 9. Wave shape of 4:1 MUX

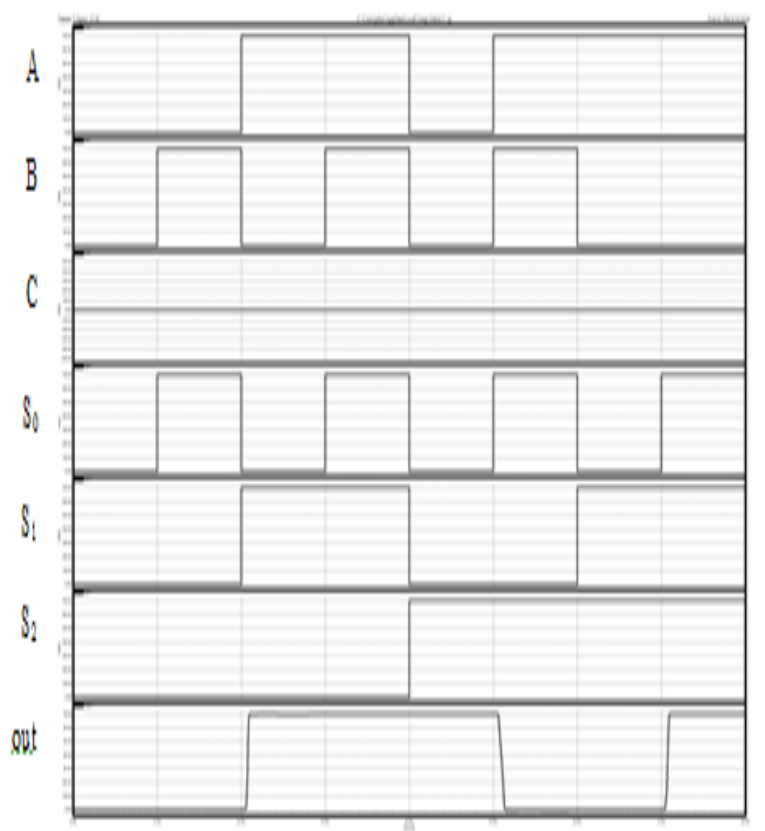

Figure:10. Wave Shape obtained for 1-bit ALU

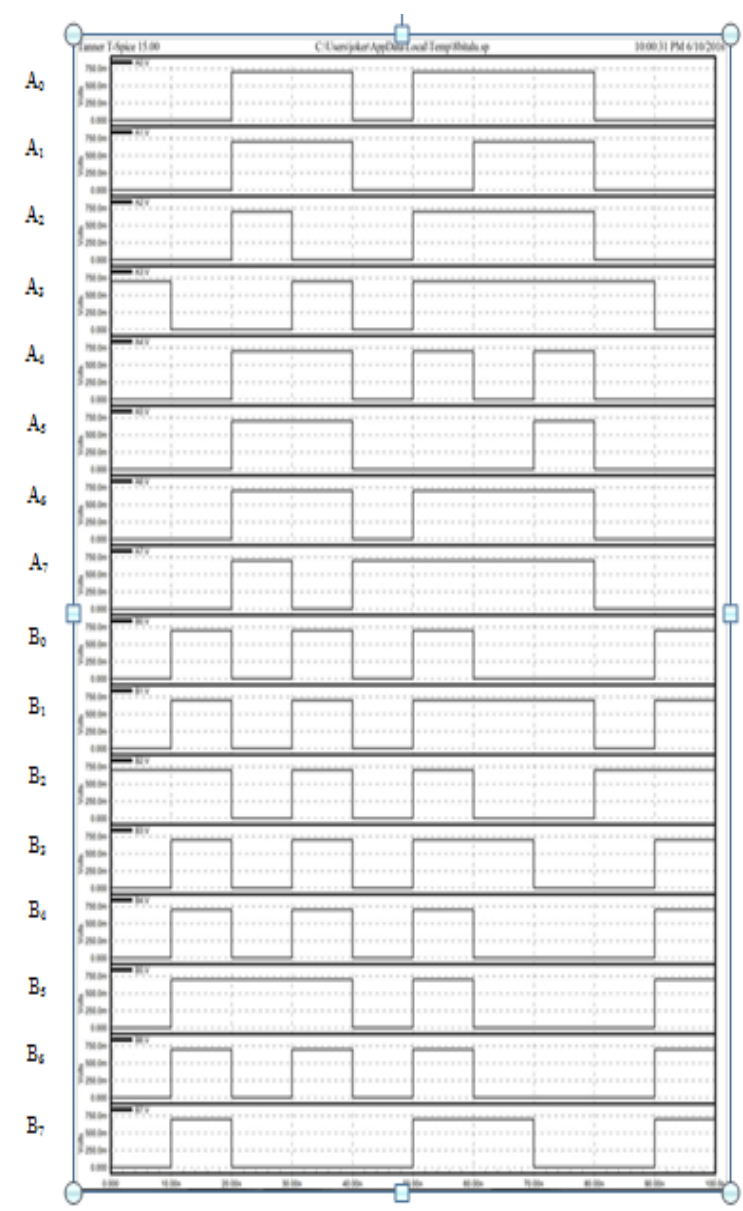

Figure 11. Input Wave shape for 8-bit ALU

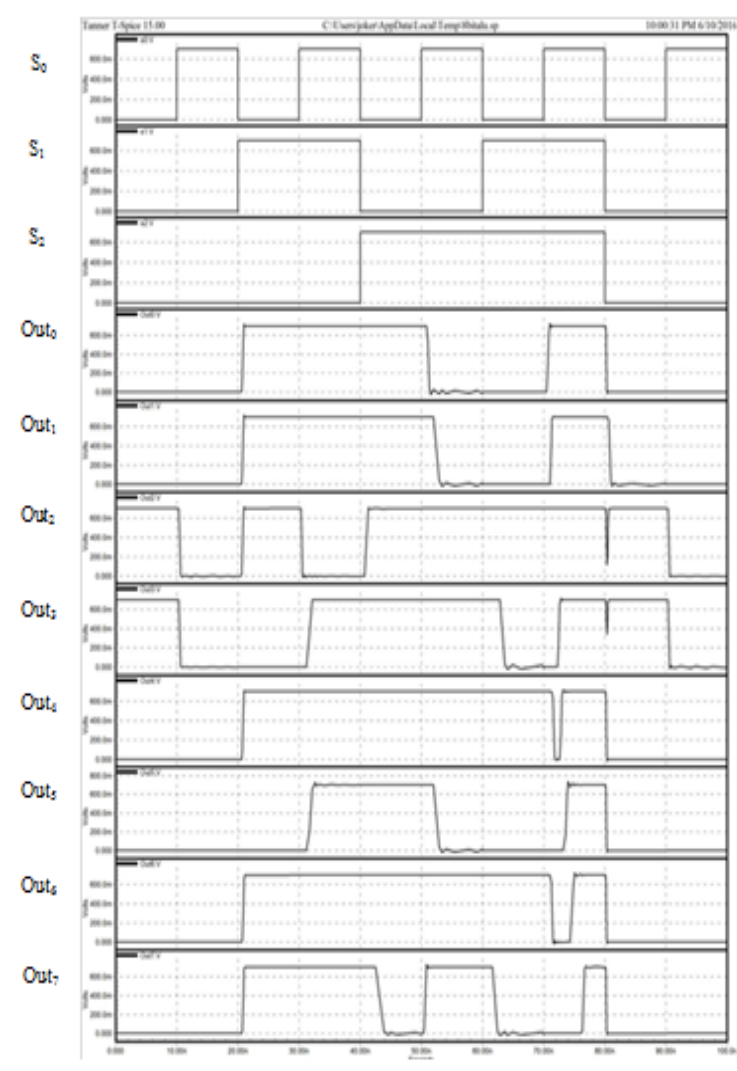

Figure 12. Select line and output wave Shape of 8-bit ALU 
Power and Delay varies with the Supply voltages. Table 2 show the results varies with supply voltage from 0.5 volt to 0.9 volt.

Table 2: Power and Delay of 8-bit ALU with the variation of $V_{D D}$

\begin{tabular}{|c|c|c|}
\hline $\mathbf{V}_{\mathbf{D D}}$ (in Volts) & Power(in $\mu \mathrm{W})$ & Delay (in nsec) \\
\hline 0.9 & 39.23 & 1.42 \\
\hline 0.8 & 25.48 & 1.49 \\
\hline 0.7 & 16.34 & 1.63 \\
\hline 0.6 & 10.12 & 1.92 \\
\hline 0.5 & 6.16 & 4.66 \\
\hline
\end{tabular}

The results of proposed model is shown in table 3 . That results iss. obtained by $0.7 \mathrm{~V}_{\mathrm{DD}}$,

Table 3: Proposed Design Results

\begin{tabular}{|l|l|l|l|l|l|}
\hline Design & $\begin{array}{l}\text { 11-T } \\
\text { FA }\end{array}$ & MUX2 & MUX4 & 1B ALU & 8B ALU \\
\hline Delay & $147 \mathrm{pS}$ & $113 \mathrm{pS}$ & $5 \mathrm{nS}$ & $10 \mathrm{nS}$ & $2 \mathrm{nS}$ \\
\hline Power & $56 \mathrm{nW}$ & $30 \mathrm{nW}$ & $514 \mathrm{nW}$ & $2 \mu \mathrm{W}$ & $16 \mu \mathrm{W}$ \\
\hline PDP & $\begin{array}{r}8232 \\
\left(n W^{*} \mathrm{pS}\right)\end{array}$ & $\begin{array}{r}3390 \\
\left(\mathrm{nW}^{*} \mathrm{pS}\right)\end{array}$ & $\begin{array}{r}2570 \\
\left(\mathrm{nW}^{*} \mathrm{nS}\right)\end{array}$ & $\begin{array}{r}20 \\
\left(\mu \mathrm{W}^{*} \mathrm{nS}\right)\end{array}$ & $\begin{array}{r}32 \\
\left(\mu \mathrm{W}^{*} \mathrm{nS}\right)\end{array}$ \\
\hline
\end{tabular}

Results comparison between previous work and purposed work shown in table 4.

Table 4 Comparison between Previous work and Purposed work

\begin{tabular}{|c|c|c|c|}
\hline Ref. No. & ALU type & Technology & Power \\
\hline 1. & FinFET based ALU & $45 \mathrm{~nm}$ & $8.38 \mathrm{~mW}$ \\
\hline 2. & $10 T$ FA based ALU & $45 \mathrm{~nm}$ & $1197.5 \mu \mathrm{W}$ \\
\hline 3. & RCA based ALU & $65 \mathrm{~nm}$ & $413.2 \mu \mathrm{W}$ \\
\hline 5. & Clock gating based ALU & $180 \mathrm{~nm}$ & $5.27 \mathrm{~mW}$ \\
\hline $\begin{array}{c}\text { Purposed } \\
\text { model }\end{array}$ & $\mathbf{8 - b i t ~ A L U}$ & $\mathbf{3 2 n m}$ & $\mathbf{1 6 . 3 4} \mathbf{\mu W}$ \\
\hline
\end{tabular}

\section{CONCLUSION}

This proposed model design for 8-Bit Arithmetic Logic Unit using 11-T FA followed the principal of GDI Techniques. Thi Paper is based on 32 Nanometer Technology. In this paper 8-b ALU operated in subthreshold region that's why consumed les power as compare with previous models. Power decreases wit $\mathrm{V}_{\mathrm{DD}}$ and Delay increases with $\mathrm{V}_{\mathrm{DD}}$. So we have selected $0.7 \mathrm{~V}_{\mathrm{D}}$ for maintain the both power as well as delay. In the propose model, less than $82 \%$ power consumed as compare with 8 -B CMOS ALU.
1. L. Dhulipalla and A. Deepak, "Design and implementation Of 4-bit ALU using FINFETS for nano scaletechnology," IEEE International Conference on Nanoscience, Engineering and Technology, pp. 190195, November 2011.

2. T. Esther Rani, M.A. Rani and R. Rao, "AREA optimized low power arithmetic and logic unit," IEEE International Conference on Electronics Computer Technology, pp. 224-228, April 2011.

3. Per Larsson-Edefors and KjellJeppson, "Timing- and Power-Driven ALU Design Training Using Spreadsheet-Based Arithmetic Exploration," IEEE 2014 10th European Workshop on Microelectronics Education (EWME), pp. 151-154, May 2014.

4. Per Larsson-Edefors and KjellJeppson, "Timing- and Power-Driven ALU Design Training Using Spreadsheet-Based Arithmetic Exploration," IEEE 2014 10th European Workshop on Microelectronics Education (EWME), pp. 151-154, May 2014.

5. Mahmoud Aymen Ahmed, M. A. Mohamed El-Bendary, Fathy Z. Amer, Said M. Singy, "Delay Optimization of 4-Bit ALU Designed in FS-GDI Technique" International Conference on Innovative Trends in Computer Engineering (ITCE’2019), pp. 534-537, Aswan, Egypt, 2-4 February 2019,

L. Raja, K. Thanushkodi and T. Hemalatha, "Comparative Analysis of Various Low Power Clock Gating Design for ALU," IEEE International Conference on Electronics and Communication Systems (ICECS), pp. 1-5, February 2014.

7. Omnia Al. Badry, M. A. Abdelghany, "Low Power 1-Bit Full Adder Using Full-Swing Gate Diffusion Input Technique" International Conference on Innovative Trends in Computer Engineering (ITCE 2018), , pp. 205-208, Aswan University, Egypt

8. Uday Panwar, KavitaKhare; Leakage Power Reduction by Adaptive Logic Cell technique in CMOS VLSI Digital circuit design;Mediterrian Journal of Electronics and Communication(MEDJEC); 10; 3; 736-740; Oct 2014 (IET INSPEC).

9. Biswabandhu Jana, Anindya Jana, SubhramitaBasak and Jamun Sing, "Design and Performance Analysis of Reversible Logic based ALU using Hybrid Single Electron Transistor," IEEE Recent Advances in Engineering and Computational Sciences (RAECS), pp. 1-4, March 2014.

10. L. Raja, K. Thanushkodi and T. Hemalatha, "Comparative Analysis of Various Low Power Clock Gating Design for ALU," IEEE International Conference on Electronics and Communication Systems (ICECS), pp. 1-5, February 2014.

11. Zahid Ali Siddiqui, Park Hui-Jong and A. Lee Jeong, "Area-Time Efficient Self-Checking ALU based on Scalable Error Detection Coding," IEEE 2013 Euromicro Conference on Digital System Design (DSD), pp. 870-877, September 2013.

12. Na Gong, Jinhui Wang and Ramalingam Sridhar, "Application-Driven Power Efficient ALU Design Methodology for Modern Microprocessors," IEEE 14thInternational Symposium on Quality Electronic Design, pp. 184-188, 2013.

13. Ajay Kumar Dadoria, KavitaKhare, Tarunkumar Gupta, Uday Panwar, "Efficient Flipped Drain GatingIntegrated with Power Gating Technique for FinFET Based Logic Circuit”, WILLEY-IJNM. 31, 5, 114, 19th April 2018. DOI: 10.1002/jnm.2344 (SCI), Online ISSN:1099-1204 IM 0.861

14. Uday Panwar, KavitaKhare; Gate Replacement with PMOS stacking for Leakage reduction in VLSI Circuits; WILLEY-IJNM; 29; 4; 565576; OCT 2015 DOI: 10.1002/jnm.2112(SCI), Online ISSN:1099-1204 IM 0.861

15. Tanesh Kumar, Bishwajeet Pandey, Teerath Das and S. M. Mohaiminul Islam, "64 Bit Green ALU Design Using Clock Gating Technique on Ultra Scale FPGA," IEEE International Conference on Green Computing, Communication and Conservation of Energy (ICGCE), pp. 151-154, December 2013.

6. Ajay Kumar Dadoria, KavitaKhare, Uday Panwar, Anita Jain "Performance Evaluation of Domino Logic Circuits for wide fan-in gates with FinFET" Microsystem Technologies, Springer. 24, 8, 3341 3348, 2018. DOI: 10.1007/s00542-017-3691-3 (SCI).ISSN 09467076 ONLINE ISSN 1432-1858 IM 1.581. 
Design of 8-bit ALU Design using GDI Techniques with Less Power and Delay

\section{AUTHORS FROFILE}

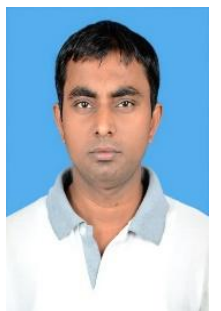

Harshmani Yadav, received Bachelor of Technology(Electronics and Communication) fromGautam Buddh Technical University,Lucknow and Master of Technology [2017-2019] pursuing fromRajiv Gandhi Proudyogiki Vishwavidyalaya Bhopal in VLSI Domain. Area of research in Design of Digital Circuit for Improved voltage usingGate diffusion input (GDI) Technique Domain.

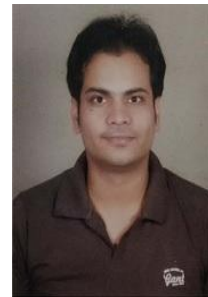

Uday Panwar, received B.E. (Electronics and Communication) in Hon's in 2006 and M.E in Hon's in 2009 with specialisation in Digital Communication, from Institute of Engineering and Technology, DAVV Indore, Ph.D. from MANIT, Bhopal. His area of interest is Design and Development of Low power High speed configuration for portable device. He has nearly 25 publications in various IEEE international conferences and SCI \& other peer reviewed journal. 